\section{New Look at Toxaemia}

SIR,-I read your leading article "New Look at Toxaemia of Pregnancy" (22 August, p. 459) with great interest and profit but would venture to make a few observations. The perinatal mortality survey was not designed to find out the incidence of pregnancy "toxaemia" and the figure of $25 \%$ is probably much too high. In Bradford during the years 1956-9 inclusive, $18 \%$ of all women delivered in the St. Luke's Maternity Hospital had a blood-pressure of or exceeding 140/90 $\mathrm{mm}$. $\mathrm{Hg}(\mathrm{H})$ on at least two occasions before the onset of labour, and about a quarter of these women also had proteinuria (HP). Since all cases of HP and the majority of cases of $\mathbf{H}$ were delivered in hospital, the incidence in the city was less than $18 \%$, more than $9 \%$, and probably of the order of $12 \%$. To use the term "toxaemia" when the B.P. is less than $140 / 90 \mathrm{~mm} . \mathrm{Hg}$ is unrealistic.

Apart from one death from eclampsia in 1956 in an unbooked and neglected case, no other maternal death between the years 1956 and 1961, inclusive, which occurred in the city was in any way associated with any form of pregnancy " toxaemia." Further, it is now agreed that no sequela following pregnancy is caused by " toxaemia."

The $18 \%$ of women in St. Luke's Maternity Hospital who had either $\mathrm{H}$ or HP might have been expected to be responsible for $18 \%$ of the perinatal mortality, whereas in fact $23 \%$ of the perinatal mortality occurred in this group of women. Many of these perinatal deaths although associated with $\mathrm{H}$ were not caused by it, and it is probably true that during the four years under review more perinatal deaths were caused by anencephaly and hydrocephaly than by hypertension.

Some of the evidence quoted in your leader might fairly be described as tendentious, and, seeing that the cause or causes of hypertension are not known, there is no reason for assuming that a raised blood-pressure during pregnancy differs from hypertension occurring in a stockbroker. Pregnancy " toxaemia" is not like the mumps or measles and does not attack at random. It picks out women with pre-existing defects and if it strikes hard before the 34 th week that woman is unlikely to have any subsequent pregnancy without H. Conversely, it is most unlikely that a woman who has a perfectly normal first pregnancy will have $\mathrm{H}$ in later pregnancies. This aspect of the problem fits in with the fact that in some areas of the world pregnancy "toxaemia" is very rare, even without antenatal care. The perinatal mortality amongst babies born to "toxaemic" women in the Tsan Yuk Hospital, Hong Kong, during the years 1960-1 amounted to but 5 per thousand total births.

The pre-existing factors which interfere with the hypothetical disturbance of amine transfer in the placenta are probably more important than the transfer itself, and it is most unfortunate that some of the workers you quote fail to define " toxaemia," to state the time of its onset, or even to indicate the ultimate fate of the infant.

I have ventured to offer some reasons as to why in my view solution of the problem of amine transfer in the placenta will not solve the problem of "toxaemia" of pregnancy. My main purpose in writirig this letter, however, is to stress the fact that we know how to prevent maternal loss and to cut foetal loss from pregnancy "toxaemia." The main problem in obstetrics is prematurity, which is often linked to " toxaemia," and it may well be that we may one day discover how Holland halted her incidence in a short period of time. -I am, etc.,

Lund, Sweden.

G. W. Theobald.

${ }_{*}^{*}$ Mr. G. W. Theobald is to be congratulated on the excellent figures achieved at St. Luke's. If the perinatal mortality report-not primarily designed as a survey of toxaemia in Britain but the best we have-points to a more gloomy picture, the discrepancy may partly be accounted for by its diagnostic criteria being less stringent than those of $\mathrm{Mr}$. Theobald. But definitions of toxaemia are apt to differ owing to the absence of a known causative agent. The leading article tried to outline some of the newer biochemical attempts to come to grips with this seemingly intractable aetiological problem.-ED., B.M.F.

\section{Cramps in Pregnancy}

SIR,-A possible answer to Dr. J. L. Fraser's query (12 September, p. 687), about pain in the right subcostal region in pregnant women is that of interchondral joint subluxation. I have described this in the Practitioner. $^{1}$ The lower margin of the rib cage is lifted up in later pregnancy both directly by the uterus and by the pull of the rectus abdominis muscle. It is assumed that the leverage on this area is greater on the right side because of the bulk of the liver. The lifting of a lower costal cartilage over an upper one strains the small interchondral joints between them, so causing pain. The joints are synovial and are present between the sixth and seventh, seventh and eighth, and the eighth and ninth costal cartilages.

Occasionally pain may be acute in this area when the woman bends down. More often it is a dull ache. Finger pressure may show the maximum point of tenderness to be just over the lower rib cage and not below it. Hyperaesthesia is not at all uncommon, perhaps because of the nearness of the intercostal nerve, and perhaps the pain may be referred away from the area of the joint itself. In bad cases local anaesthesia introduced into the joint relieves the pain immediately. In one case where the pain was excruciating during labour $I$ infiltrated the intercostal nerve just lateral to the sacrospinalis muscle mass with immediate relief. The pain in this instance was on the left side and had led to a diagnosis of cardiac ischaemia.

Of course I am not suggesting that Dr. Fraser's explanation of pain in the subcostal region as possibly due to biliary disorder is untrue ; only that interchondral subluxation also may cause discomfort in much the same place.-I am, etc.

\section{Department of Gynaecology, \\ t. Thomas's Hospital Medical School London S.E.1. \\ Reference \\ 1 Rhodes, P., Practitioner, 1958, 181, 304.}

\section{Treatment of Thyroid Disorders}

SIR,-We were very interested in the symposium on the treatment of thyroid disorders (22 August, p. 493). In the endocrine department at the New End Hospital 800 new patients with thyroid disorders are seen and over 300 thyroidectomies are performed per annum. A third of the thyroidectomies are for thyrotoxicosis. We have combined medical, surgical, and radiotherapy units working in close co-operation with each other, ${ }^{1-3}$ and in this letter we briefly summarize our experience with the surgical treatment of thyrotoxicosis which we hope will be of some value.

Of all the thyrotoxic patients over twothirds are treated by means of partial thyroidectomy. In the last ten years about 1,100 partial thyroidectomies have been performed on thyrotoxic patients. The mortality has been less than $0.1 \%$, the incidence of transient hypoparathyroidism was $1.7 \%$ and permanent hypoparathyroidism less than $0.4 \%$. There were no cases of bilateral recurrent laryngeal nerve damage. The incidence of unilateral recurrent laryngeal nerve damage was $1.9 \%$, more than half of these recovering completely within a few months. The overall incidence of hypothyroidism was about $8 \%$, but the majority of these cases showed lymphadenoid infiltration in the thyroid gland on histological examination and, therefore, most likely would have become hypothyroid whatever form of treatment was instituted. There appears to be no increased hazard to the pregnant patient undergoing thyroidectomy, provided the thyrotoxicosis has been controlled by anti-thyroid drugs and operation undertaken between the twelfth and twenty-fourth week of pregnancy. Of 75 partial thyroidectomies performed on pregnant patients there was not a single foetal loss and there were no adverse effects on the mother. The occurrence of hypothyroidism or a goitre was not reported in any infant.

The main disadvantage of drug treatment is the very high relapse rate of $60 \%$ except for the under-25 age group.

Out of $\mathbf{4 0 0}$ thyrotoxic patients treated with a therapeutic dose of radioactive iodine the incidence of hypothyroidism was $20 \%$ at one year and $40 \%$ at five years. About $20 \%$ of the patients needed more than one treatment dose of ${ }^{131} \mathrm{I}$. Hypoparathyroidism did not occur in a single case.

In the experience in this hospital partial thyroidectomy has a negligible mortality and a very low morbidity and relapse rate. We conclude that for the majority of thyrotoxic patients partial thyroidectomy, provided it is performed by a surgeon experienced in thyroid surgery, offers the best possible results. Drug treatment and radio-iodine have a valuable role to play in special circumstances.-We are, etc.,

$\begin{array}{ll}\text { New End Hospital, } & \text { W. Singer. } \\ \text { London N.W.3. } & \text { J. E. PIERCY. }\end{array}$

REFERENCES

Greene, R., 7. Endocrinol., 1950, 7, 1

2 Lange, M. J., Proc. roy. Soc. Med., 1961, 54,

\section{Treatment of Recurrent Herpes Simplex}

SIR,-It seems a pity that your contributor in a leading article (5 September, p. 587) should have taken a relatively pessimistic view of the efficacy of 5-iodo-2-deoxyuridine in the treatment of herpes simplex. While it does appear that this substance has no effect on the recurrence rate of these lesions, there have been a number of other reports ${ }^{1-3}$ besides 